www.cya.unam.mx/index.php/cya

Contaduría y Administración, 65 (5), Especial COVID-19, 2020, 1-19

\title{
Impacto de la crisis económica por COVID-19 en el sistema de pensiones mexicano y perspectivas ante el proyecto de su reforma
}

\author{
Impact of the economic crisis caused by COVID-19 on the Mexican pension system \\ and prospects considering its reform Project
}

\author{
Marissa R. Martínez-Preece*, \\ Carlos Zubieta-Badillo
}

Universidad Autónoma Metropolitana, México

Recibido el 11 de octubre de 2020; aceptado el 19 de diciembre de 2020

Disponible en Internet el: 28 de diciembre de 2020

\section{Resumen}

Considerando el actual entorno económico y financiero, el objetivo de este trabajo es determinar los efectos de la crisis económica, provocada por la pandemia de COVID-19, en el riesgo del sistema de pensiones mexicano y su impacto ante la propuesta de reforma del sistema. Para alcanzar este objetivo se analizó el comportamiento del riesgo del sistema a través de su volatilidad condicional, la cual se estimó utilizando modelos de la familia GARCH. Por otra parte, se evalúo la pertinencia de los cambios en las aportaciones y el tiempo de cotización propuestos por el proyecto de reforma del sistema de pensiones, ante el impacto de la crisis en los ingresos de los trabajadores. Lo anterior se realizó simulando con el método de Monte Carlo el ahorro total acumulado al final de la vida activa de un trabajador tipo suponiendo diversos niveles de ingreso. Se concluyó que se presentó persistencia de la volatilidad y efectos de asimetría, y que los trabajadores estarán en una mejor posición si la reforma incluye medidas que incrementen sus aportaciones pero sin disminuir el tiempo de cotización.

Código JEL: C58, G23, J26

Palabras clave: Sistemas de pensión; SIEFORE; Volatilidad condicional; crisis económica por COVID-19

\footnotetext{
${ }^{*}$ Autor para correspondencia Correo electrónicomrmp@azc.uam.mx (M. Martínez Preece).

La revisión por pares es responsabilidad de la Universidad Nacional Autónoma de México.

http://dx.doi.org/10.22201/fca.24488410e.2020.3101

0186- 1042/@ 2019 Universidad Nacional Autónoma de México, Facultad de Contaduría y Administración. Este es un artículo Open Access bajo la licencia CC BY-NC-SA (https://creativecommons.org/licenses/by-nc-sa/4.0/)
} 


\begin{abstract}
In view of the current economic and financial environment, the aim of this paper is to determine the effects of the economic crisis caused by the COVID-19 pandemic, on the Mexican pension system risk, and its impact on the project that has been proposed for it. To achieve this goal, the risk behavior of the system was analyzed through its conditional volatility estimated by GARCH family models. The relevance of the changes stated in the reform project regarding the amount and length of the contributions made by the workers, in the light of the impact that the crisis will have on their salaries, was evaluated. This was done by simulating with the Monte Carlo method the total savings balance accumulated at the end of the active life of a standard worker, assuming different income levels. It was concluded that persistence of volatility and asymmetry effects were present, and that workers would be better off if the reform includes measures that increase the amount of the contributions but do not decrease the contribution period.
\end{abstract}

JEL code: $C 58$, G23, J26

Keywords: Pension systems; SIEFORE; Conditional volatility; economic crisis by COVID-19 pandemic

\title{
Introducción
}

A 23 años de su operación, cada día está más próximo el momento en que el sistema de pensiones de contribuciones definidas deba probar si ha sido suficiente para ofrecer a los trabajadores jubilados una pensión por encima del mínimo de subsistencia. El periodo de transición entre el esquema de jubilación anterior, de beneficios definidos, y el actual está próximo a terminar, debido a la proximidad de la fecha de retiro de aquellas personas que hayan cotizado de manera continua, durante toda su vida laboral, 1250 semanas, bajo el sistema de contribuciones definidas que se introdujo en México en julio de 1997.

Bajo el esquema actual de jubilaciones, el monto de la pensión depende no sólo de las aportaciones y el tiempo durante el cual se realicen, sino también de los rendimientos que puedan obtener los recursos depositados en las cuentas individuales de los trabajadores que se canalizan a los mercados financieros. Por lo tanto, es necesario que se cuente con condiciones económicas y financieras estables.

El 11 marzo de 2020, el brote de coronavirus que inició en diciembre en la Ciudad de Wuhan, en la República Popular China, fue oficialmente declarado como pandemia por la Organización Mundial de la Salud, OMS. Organismos internacionales como la Organización para la Cooperación y Desarrollo Económico, OCDE, el Fondo Monetario internacional, FMI, el Banco Mundial, BM coincidieron en que la pandemia ocasionada por el COVID-19 ha provocado una severa crisis económica que afectará a todas las regiones del mundo. Sus efectos se pronostican como graves, y que tendrán consecuencias en el sector salud y en la economía global, con alcances a nivel social.

Ante este panorama, el sistema de pensiones en México desempeña un doble papel, por un lado, ha sido afectado por la crisis, pero por otra, el actual sistema pensionario pudiera convertirse en un costo oculto para el presupuesto público contribuyendo al incremento del déficit fiscal.

Considerando el actual entorno económico y financiero, el objetivo de este trabajo es determinar los efectos de la crisis económica, provocada por la pandemia de COVID-19, en el riesgo del sistema de pensiones mexicano, y su impacto ante la propuesta de reforma del sistema. Se partió de los supuestos que la crisis económica elevará los niveles de riesgo del sistema pensionario del país, y que provocará un aumento en el desempleo, afectando el nivel de ingresos de los trabajadores y, por lo tanto, impactando en el total que podrían acumular al final de su vida activa. La contribución de este trabajo yace en el análisis de los efectos inmediatos de la crisis económica provocado por la pandemia en el riesgo del sistema pensionario y en la determinación de la pertinencia de los principales puntos que incluye la reforma ante el impacto de la crisis en los ingresos de los trabajadores. Considerando lo anterior, se tratará el impacto de la crisis por COVID-19 en los tres puntos que permiten que los trabajadores acumulen recursos en sus cuentas individuales: las aportaciones, el tiempo de cotización y la influencia de los mercados financieros en los fondos de pensión. 


\section{M. del R. Martínez-Preece y C. Zubieta-Badillo / Contaduría y Administración 65(5) Especial COVID-19, 2020, 1-19 http://dx.doi.org/10.22201/fca.24488410e.2020.3101}

Para conseguir el objetivo, este trabajo se dividió en seis partes incluyendo la introducción. En la segunda sección se hace una revisión de la literatura. En la tercera parte se presenta el entorno económico nacional e internacional. En la cuarta sección se presenta la metodología utilizada para determinar la dinámica del riesgo del sistema de pensiones y la pertinencia de la propuesta de reforma considerando las aportaciones y el tiempo de cotización. En la siguiente sección se presentan los resultados de los modelos GARCH para determinar la volatilidad condicional y de la simulación de los ahorros totales acumulados por los trabajadores. En la última parte se ofrecen las conclusiones.

\section{Revisión de la literatura}

Los mercados financieros internacionales han incrementado su relación con los sistemas pensionarios desde hace tres décadas debido a que sus sistemas financieros canalizan recursos de los trabajadores hacia ellos. Lachman (2013) hace un estudio de los distintos esquemas de pensiones. En su trabajo señala el desempeño de los distintos tipos de sistemas pensionarios y encuentra que todos han sido afectados durante períodos de alta volatilidad, y en especial a raíz de la crisis económica de 2008. Los esquemas de beneficios definidos han sido susceptibles a los déficits fiscales hecho que, aunado a problemas demográficos, ha desencadenado una falta de sustentabilidad a largo plazo, y los de contribuciones definidas han sido afectados directamente por la volatilidad de los mercados financieros. Ambos tipos de esquemas pensionarios han experimentado bajos rendimientos e incluso pérdidas severas en períodos de crisis.

Por su parte, Ortiz et al. (2019) analizaron el sistema de pensiones de capitalización a nivel internacional, centrándose en aquellos que han regresado a un sistema de pensiones de beneficios definidos después de haber aplicado sistemas de contribuciones definidas en sus países. De 30 países que entre 1981 y 2014 privatizaron total o parcialmente sus sistemas de pensiones, 18 de ellos revirtieron parcial o totalmente la privatización de sus pensiones, al hacerse evidente las fallas del sistema de cuentas individuales después de la crisis de 2008. Entre los problemas que presentaron se destacan: altos costos fiscales y administrativos, transferencia de riesgos de los mercados financieros a los trabajadores, estancamiento o disminución de las tasas de cobertura, deterioro de los montos en las pensiones recibidas y profundización de las desigualdades de género e ingreso.

Los problemas de sustentabilidad y sostenibilidad de los sistemas de reparto han sido ampliamente analizados y debatidos desde finales de la década de los ochenta. No obstante, Encinas-Goenechea et al., (2020) vuelven a analizar este tipo de esquema de pensiones ante la luz de la realidad económica que se ha vivido en los últimos años, centrándose en el caso de las pensiones de los países que conforman la Unión Europea. Los autores estudian cómo factores demográficos se unen a un descenso de la actividad financiera y económica que genera obstáculos para mantener bajas tasa de desempleo y propiciar el crecimiento, lo cual impone un gran peso para lograr la sustentabilidad de los sistemas públicos de pensiones. En busca de solucionar este problema los autores presentan las medidas tomadas por los países de la mencionada región para conseguir sistemas sustentables.

Para el caso del sistema de pensiones de México y su desempeño, la literatura es aún escasa. Hay un número reducido de investigaciones que analizan el comportamiento del esquema pensionario de capitalización mexicano ante una crisis económica. Las bajas tasas de reemplazo han sido una preocupación desde la crisis de 2008. A partir de ésta, se hizo evidente que la tasa de reemplazo ${ }^{1}$ que ofrece el actual sistema de pensiones no alcanzará el $60 \%$ que se obtenía bajo el sistema de reparto. En un estudio realizado sobre la situación de las pensiones en México, (OCDE, 2016), se enfatizó que

\footnotetext{
${ }^{1}$ La tasa de reemplazo se refiere al porcentaje del salario que recibe un trabajador retirado respecto al sueldo devengado antes de su retiro.
} 


\section{M. del R. Martínez-Preece y C. Zubieta-Badillo / Contaduría y Administración 65(5) Especial COVID-19, 2020, 1-19 http://dx.doi.org/10.22201/fca.24488410e.2020.3101}

se deben aplicar medidas que aumenten el nivel de las cotizaciones para alcanzar al menos una tasa de reemplazo del $50 \%{ }^{2}$ y, con este mismo objetivo, se incremente el tiempo de cotización para tener derecho a una pensión.

En su investigación Ramírez (2019), siguiendo la línea de análisis de Ortiz et al. y tomando como referencia los indicadores de la Organización Internacional del Trabajo, analiza para el esquema de pensiones mexicano la propuesta de regresar a un sistema público de pensiones. La autora argumenta que el regreso a un sistema de reparto estaría justificado debido a que el sistema de contribuciones definidas no ha ampliado su cobertura y a que la tasa de reemplazo es más baja que la ofrecida por el sistema de reparto. Asimismo, expone que se ha ampliado la desigualdad de ingresos entre géneros y que se han generado altos costos de transición, entre un sistema y otro, incrementando las presiones fiscales.

De la Torre-Torres et al. (2020a) encuentran que el índice de rendimiento neto que ofrece la Comisión Nacional del Sistema de Ahorro para el Retiro, CONSAR, no es un buen indicador en época de crisis y proponen un modelo markoviano de cambio de régimen que ofrece más información para la toma de decisiones sobre la política de inversión de los fondos de pensión.

Dentro de la literatura dedicada a la creación de indicadores para el caso mexicano, algunas de estas investigaciones tratan de la creación de índices que van más allá de sólo mostrar el comportamiento de los fondos de pensión al tener como objetivo medir su desempeño. Núñez y León (2019) proponen un portafolio de referencia (benchmark) de tal forma que se optimice la política de inversión de los fondos de pensión de México, con el objetivo de alcanzar la máxima tasa de reemplazo posible. Como resultado obtienen un portafolio más conservador que los que actualmente manejan las SIEFORE Básicas, integrado por un bajo porcentaje de activos de riesgo y con activos de corto plazo con vencimientos entre tres y cinco años.

De la Torre-Torres et al. (2015a) y De la Torre-Torres et al. (2015b), diseñan dos indicadores de referencia. En su primer artículo usan un portafolio de mínima varianza y en el segundo presentan un indicador de referencia llamado Actual Position Benchmark (APB) basado en el ciclo de vida del trabajador. En la práctica, tomando como referencia el último índice, S\&P Dow Jones/BMV México lanzó al mercado benchmarks para el sistema de pensiones mexicano.

La volatilidad de los rendimientos de los fondos de pensión en México, es estudiada bajo dos escenarios diferentes con cambio de régimen en los trabajos de Martínez-Preece, Sosa y Zubieta (2019) y de López-Herrera, Martínez-Preece y Santillán-Salgado (2020).

\section{Entorno económico nacional e internacional}

Ante la crisis económica internacional varios organismos internacionales han formulado pronósticos sobre los efectos de la pandemia en la economía mundial. El FMI (2020) pronostica que esta crisis es la más severa después de la gran depresión, con una caída del PIB mundial a $-4.5 \%$ para 2020, después de haber presentado un crecimiento de $2.9 \%$ en 2019 y un pronóstico de recuperación para 2021 que alcanzará 5.3\% ${ }^{3}$. En la Figura 1 se observa como México, Estados Unidos, los países de la zona del euro y el mundo tendrán una caída negativa de su producción durante este año. La excepción será China que en septiembre de 2020 empezó su recuperación y se considera que alcanzará un $1 \%$ de crecimiento en el PIB en este año y de $8.2 \%$ en 2021.

\footnotetext{
${ }^{2}$ Se calculó que una tasa de reemplazo del 50\% se alcanza, con una probabilidad del 75-90\%, con una cotización media entre 13 al $18 \%$, durante 40 años.

${ }^{3}$ Datos a septiembre de 2020.
} 


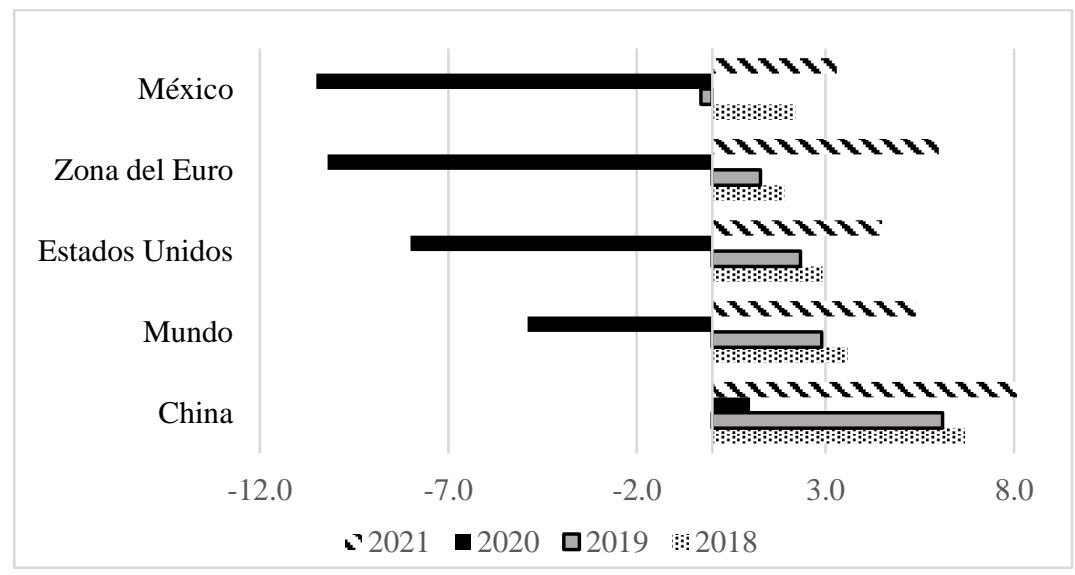

Figura 1. Caída porcentual del PIB

Fuente: elaboración propia con datos del FMI (2020)

Las proyecciones para el comercio internacional también prevén una importante caída durante 2020, debido principalmente a la ruptura de las cadenas de suministros, FMI (2020). Se proyecta que en 2020, el comercio internacional tenga un crecimiento negativo de $-11.9 \%$, con una recuperación en 2021 de $8.0 \%$. La demanda de petróleo se redujo un $55 \%$ durante el primer semestre de este año debido a la pandemia, por lo que se verán afectados negativamente los ingresos de los países exportadores de petróleo. Otro sector que desde el principio ha sufrido los efectos de la crisis económica es el turismo. Las medidas de mitigación y aislamiento, durante 2020, han provocado cierres temporales del $100 \%$ de destinos turísticos. En un escenario moderado (UNCTAD, 2020) se espera que se pierda un tercio de los ingresos turísticos en 2020.

Respecto a las tasas de desempleo, los países de la OCDE (2020a), reportaron importantes incrementos en este rubro durante los primeros meses de 2020, reportando $42.59 \%$ en julio de 2020.

Dada la situación actual provocada por la pandemia de COVID-19, el volumen total de los activos administrados por los fondos de pensiones de los países miembros de la OCDE (2020b) alcanzaron USD 29,757,3354 millones, lo cual representa una contracción del 6\% al comparar el primer trimestre de 2020 con la misma fecha de 2019, debido al descenso de los mercados bursátiles ante el anuncio de la pandemia.

El panorama en México es similar al internacional. El crecimiento del PIB en el segundo trimestre del 2020 tuvo una caída sin precedente, incluso comparado con crisis anteriores, como se aprecia al revisar el mismo período en 1995 y 2009, éste último consecuencia de la crisis de 2008. En la Figura 2 se observa que la variación del PIB llegó a -18.68\% en el segundo trimestre de este año, comparada con el $-9.68 \%$ en el segundo trimestre de 1995 y $-8.93 \%$ en el mismo período en 2009. Esta baja en el PIB fue consecuencia del descenso del consumo interno que llevó a una caída en la actividad industrial y ésta a un incremento en el desempleo.

\footnotetext{
${ }^{4}$ Cifra proyectada para 2020.
} 


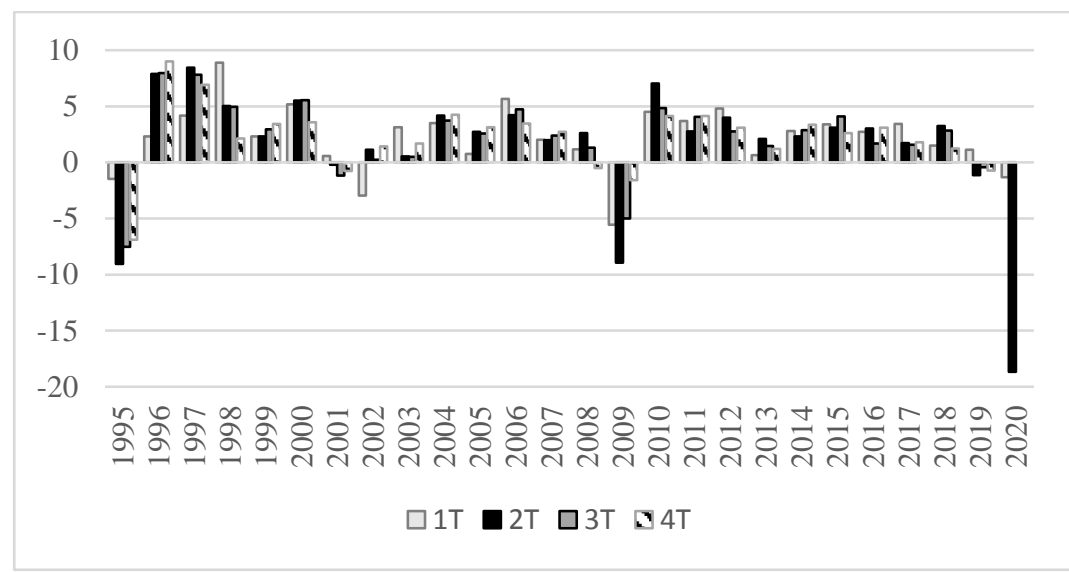

Figura 2. Variación del Producto Interno Bruto. (Valores constantes en millones de pesos a precios de 2013) Fuente: elaboración propia. INEGI

Los índices de consumo privado y el índice de producción industrial presentaron fuertes caídas en el primer semestre de 2020, con variaciones negativas del $23.52 \%$ y $30.08 \%$, respectivamente, producto de las medidas del confinamiento que se empezaron a aplicar a finales de marzo de 2020.

La falta de crecimiento en el consumo y en la producción industrial se reflejó en la tasa de desempleo, de subocupación y de condiciones críticas de ocupación. La tasa de desocupación presentó un incremento en el segundo trimestre de 2020 con respecto al primero, al alcanzar 5.34\% en el segundo trimestre comparado con el $3.26 \%$ al cierre del primer trimestre. La tasa de subocupación se incrementó de forma importante en el segundo trimestre, llegando al $20.5 \%$ comparado con el $8.65 \%$ en el primer trimestre. La tasa de condiciones críticas de ocupación alcanzó al cierre del primer trimestre 22.6\%, véase Figura 3.a) y 3.b). La tasa de informalidad laboral representó el 56.2\% de la población mayor a 15 años, según reportó INEGI al cierre de marzo de 2020.

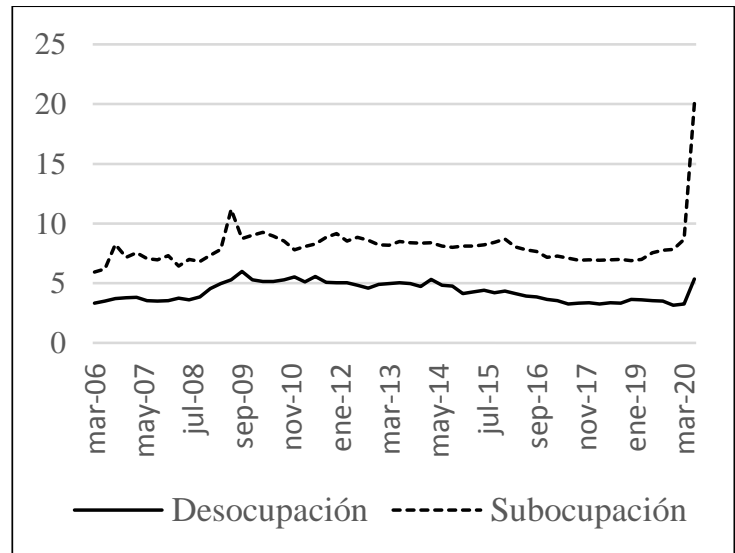

Figura 3.a) tasa de desocupación ${ }^{5}$ y subocupación ${ }^{6}$.

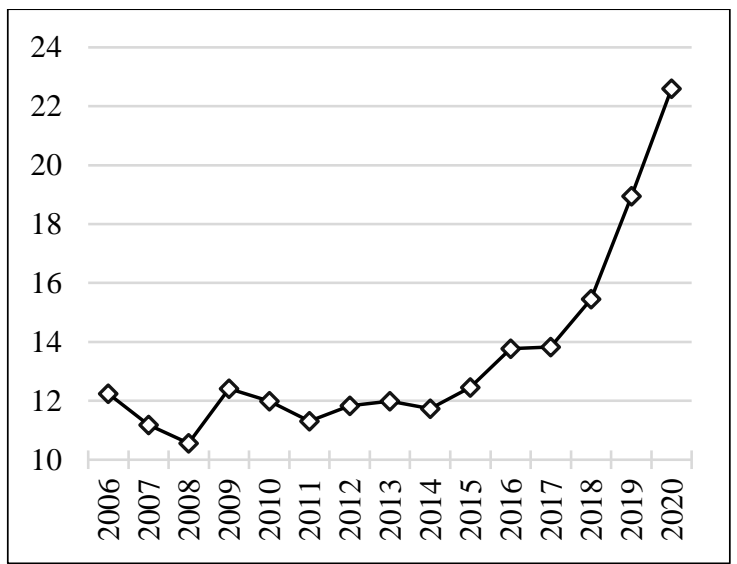

Figura 3.b) tasa de condiciones críticas de ocupación ${ }^{7}$ Figura 3. Mercado laboral

Fuente: elaboración propia con datos de INEGI

\footnotetext{
${ }^{5}$ La tasa de desocupación considera a la población que se encuentra sin trabajar, pero que está buscando trabajo. Serie desestacionalizada como porcentaje de la población económicamente activa.

${ }^{6}$ Tasa de subocupación, es el porcentaje de la población ocupada que tiene la necesidad y disponibilidad de ofertar más tiempo de trabajo de lo que su ocupación actual le permite, como porcentaje de la población económicamente activa.

${ }^{7}$ La tasa de condiciones críticas de ocupación incluye a las personas que se encuentran trabajando menos de 35 horas a la semana por razones ajenas a sus decisiones, más las que trabajan más de 35 horas semanales con ingresos mensuales inferiores al salario mínimo y las que laboran más de 48 horas semanales ganando hasta dos salarios mínimos, como porcentaje de la población ocupada.
} 
Respecto al contexto financiero de México, la tasa de fondeo gubernamental disminuyó de $7.28 \%$ el $1^{\circ}$ de enero de este año a $4.51 \%$ el 30 de agosto de 2020. Esta tasa de fondeo empezó a disminuir desde finales de 2019, aunque esta tendencia se hizo más pronunciada a partir de la pandemia, y ha seguido la misma tendencia a la baja que la tasa de fondos de la Reserva Federal de Estados Unidos, misma que presentó un nivel de 0.10\% al cierre de agosto de 2020.

Los mercados bursátiles también presentaron una disminución durante este año. El IPyC reportó una fuerte disminución del $21.56 \%$ entre enero y marzo de este año, sin embargo, ésta representó menos de la mitad de la que sufrió durante la crisis del 2008. En esa ocasión, de mayo de 2008 a febrero de 2009, el IPyC tuvo una pérdida del 44.48\%. En la Figura 4.a) se muestra el comportamiento del Índice de Precios y Cotizaciones, el cual pasó de 44,108.31 a 34,554.59 puntos con un ligero aumento a 36,840.73 puntos en agosto. El IPyC mantuvo una tendencia a la baja, aunque con una ligera recuperación en el segundo trimestre del año, a diferencia del Índice Mundial MCSI que presentó una disminución en abril, pero se recuperó rápidamente logrando alcanzar los niveles que tenía antes de la crisis a finales de mayo, véase Figura 4.b).

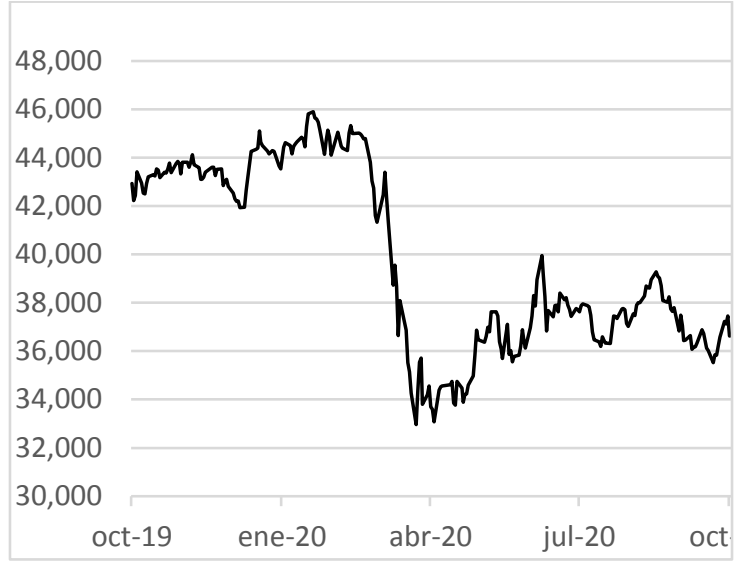

Figura 4.a) Índice de Precios y Cotizaciones

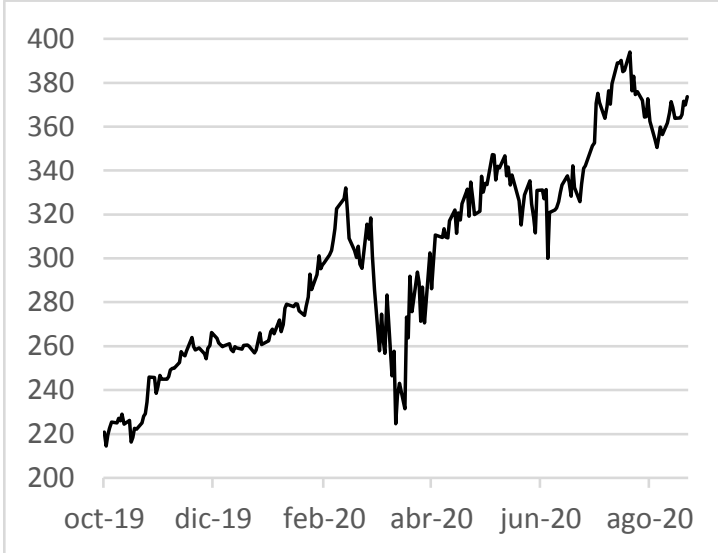

Figura 4.b) MSCI World Index

Figura 4. Índices bursátiles

Fuente: elaboración propia

El mercado cambiario presentó una depreciación con respecto al dólar estadounidense de $28 \%$ ante el anuncio de la pandemia por COVID-19, sin embargo, aunque fue una caída severa su intensidad fue menor a la depreciación que sufrió entre septiembre y octubre de 2008, misma que alcanzó el 36\%. En 2020 la recuperación ha sido más rápida que en 2008, véase Figura 5.a).

Por otra parte, la deuda pública, que ya mostraba una tendencia al alza desde 2017, se incrementó en un $13.01 \%$ de enero de 2020 a julio de 2020, véase Figura 5.b). 
M. del R. Martínez-Preece y C. Zubieta-Badillo / Contaduría y Administración 65(5) Especial COVID-19, 2020, 1-19 http://dx.doi.org/10.22201/fca.24488410e.2020.3101

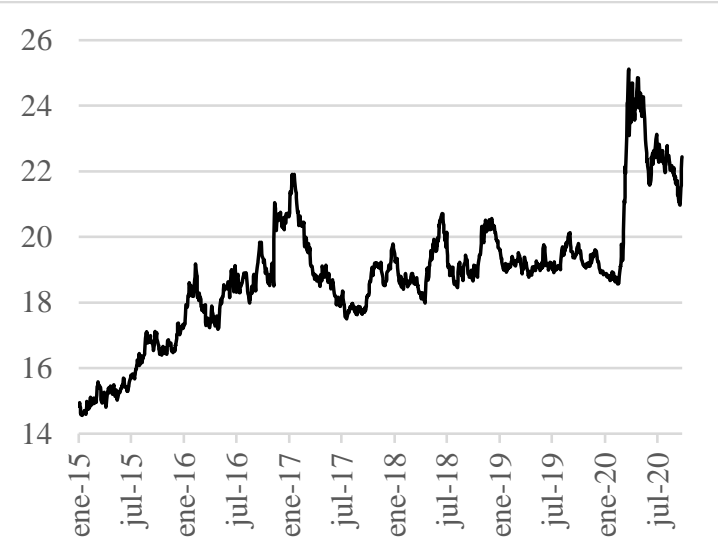

Figura 5.a) Tipo de cambio del peso mexicano frente al dólar de Estados Unidos

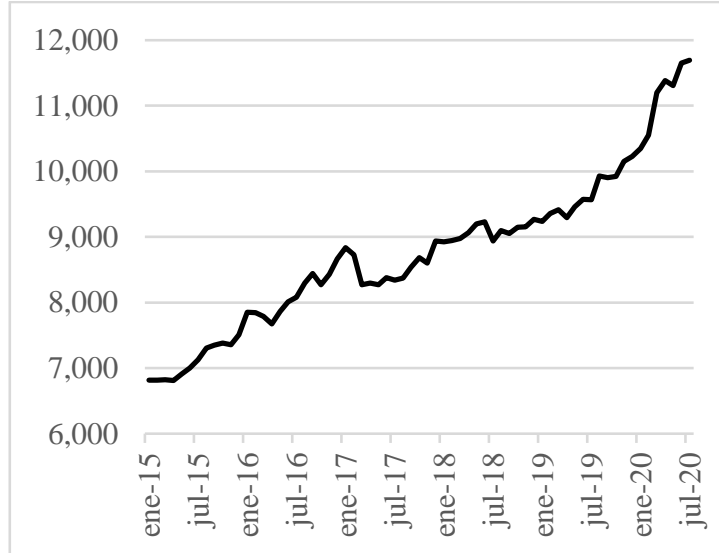

Figura 5.b) Deuda pública (en miles de millones de pesos)

Figura 5. Tipo de cambio MXN-USD y volumen de la deuda pública.

Fuente: elaboración propia con datos de BANXICO

Uno de los impactos de la baja en las tasas de interés y de la caída en el producto, con su consecuente efecto negativo en el mercado bursátil mexicano, es que durante los primeros siete meses del año los instrumentos de renta fija gubernamental y de renta variable en tenencia de no residentes presentaron una variación negativa, debido a la salida de capitales durante los primeros meses del año. El volumen de títulos de renta fija en tenencia de no residentes de enero a julio de este año se redujo en $26.56 \%$ y la disminución en la tenencia de instrumentos de renta variable por no residentes cayó un $28.35 \%$ (Banxico, 2020).

\section{Panorama del sistema de pensiones}

En un intento por permitir que los trabajadores reciban mayores rendimientos y que su ahorro no esté expuesto a un riesgo innecesario se reestructuró la política de inversión mediante la creación de nuevos tipos de SIEFORE. Las SIEFORE básicas generacionales, SBG, empezaron a funcionar el 14 de diciembre 2019 y consisten en: un fondo para trabajadores de 25 años o menores, 8 fondos con rangos de 5 años de edad, iniciando con trabajadores mayores de 25 años y un fondo de pensión para trabajadores de 65 años o mayores. Los activos que integran los portafolios de las SIEFORE varían con el tiempo de acuerdo con la edad de los trabajadores. Los límites de inversión en activos de riesgo son más altos para los fondos destinados a los trabajadores más jóvenes, y activos de renta fija se incorporarán en mayores proporciones a los fondos de pensión para trabajadores de mayor edad. La figura 6.a) muestra la proporción de activos que manejaban los tipos de SIEFORE antes de la creación de las $\mathrm{SBG}^{8}$, y la 6.b) la proporción de activos con que empezaron a funcionar las SBG.

\footnotetext{
${ }^{8}$ Información al cierre de noviembre de 2019.
} 


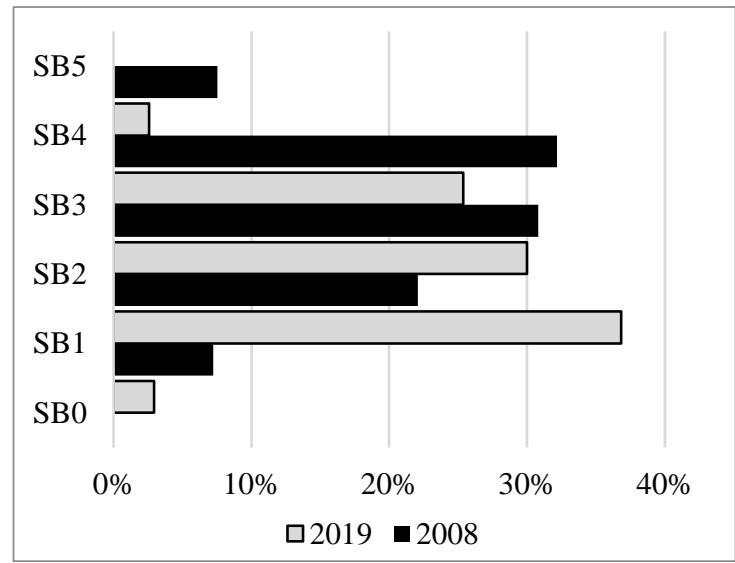

Figura 6.a) Activos manejados por la SIEFORE Básicas.

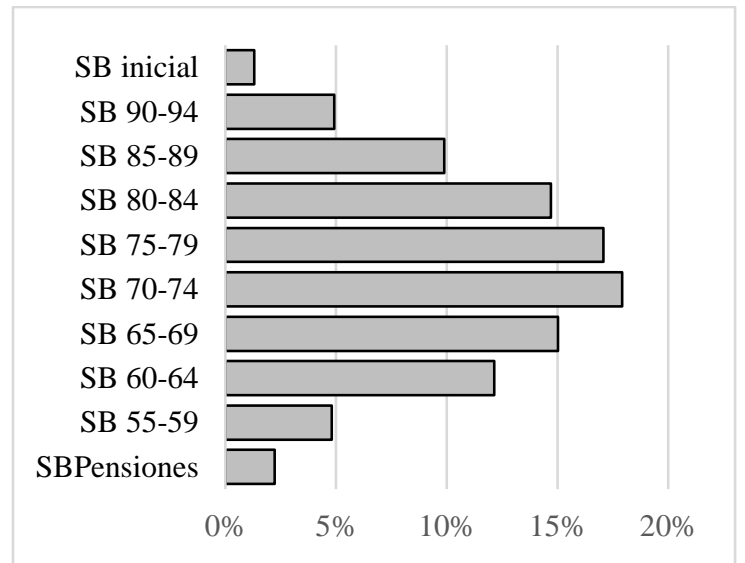

Figura 6.b) Proporción de activos manejados por las SIEFORE básicas generacionales.

Figura 6. Tipos de SIEFORE Básicas

Fuente: elaboración propia con datos de CONSAR

Con la restructuración de los fondos de pensión, la proporción de instrumentos de deuda disminuyó ligeramente, al pasar de $73.30 \%$ a $68.90 \%$, y se incrementó la proporción de instrumentos de renta variable internacional, y en menor medida de los productos estructurados y mercancías en los fondos de pensión, véase Figura 7.

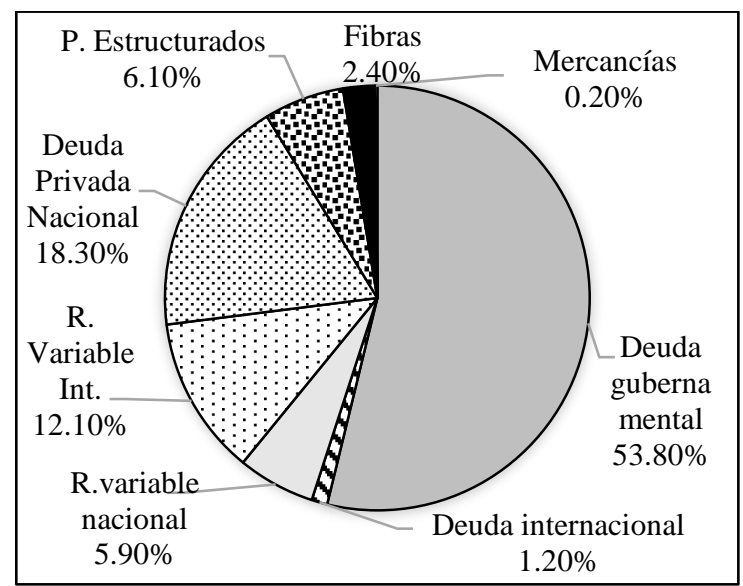

Figura 7.a) Composición de los fondos de inversión (junio 2019)

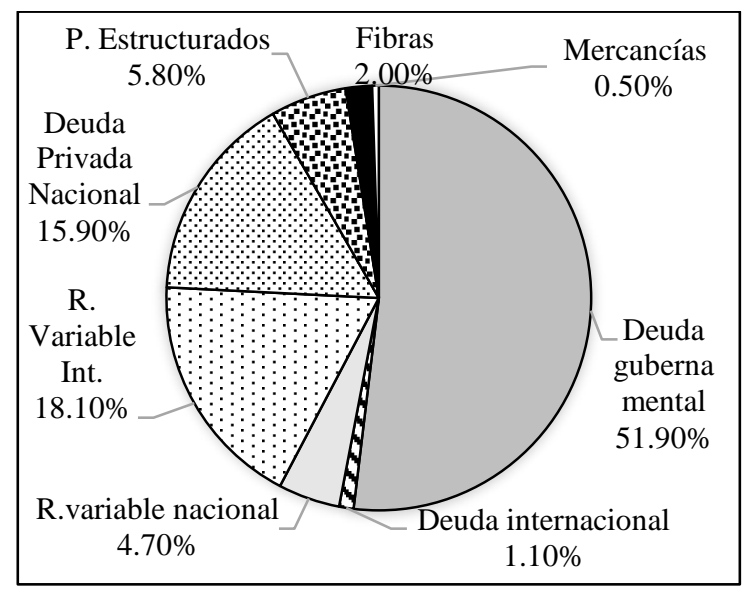

Figura 7.b) Proporción de activos manejados por las SBG (diciembre 2019).

Figura 7. Composición de los fondos de inversión. Fuente: elaboración propia con datos de CONSAR

El volumen de recursos totales manejados por las SIEFORE, a diferencia de los fondos de pensiones internacionales, no ha sufrido una disminución por la crisis, e incluso al cierre del segundo trimestre de 2020 presentó un incremento del $6.76 \%$. Esta recuperación es resultado del incremento en los precios de los instrumentos de renta fija debido a la baja en las tasas de interés y a la recuperación de los mercados bursátiles internacionales, considerando que $18.1 \%$ de los fondos de pensión están integrados por valores de renta variable internacional, y sólo $4.7 \%$ en renta nacional. Desde hace dos años, una cuarta parte de la deuda gubernamental está incorporada a los portafolios de la SIEFORE. Ante la situación actual de la salida de capital, las SIEFORE se han convertido en uno de los principales 
receptores de deuda gubernamental, a pesar de los intentos de CONSAR por diversificar los fondos de pensión y flexibilizar la política de inversión.

\section{Metodología}

Después de efectuar una revisión del entorno económico nacional e internacional y del panorama del sistema de pensiones, se modeló la volatilidad condicional de los rendimientos diarios del sistema de pensiones en su conjunto, a partir de los precios diarios de las SIEFORE Básicas registrados en la Bolsa de Valores, del 2 de julio de 2007 al 29 de junio de 2020 . Los rendimientos se calcularon como los logaritmos de las primeras diferencias del índice del sistema de las SIEFORE Básicas Generacionales. Dado que en noviembre de 2019, CONSAR (2020c) dejó de publicar los índices de cada SIEFORE y el índice del sistema en general se construyó un índice de precios simple del sistema de SIEFORE Básicas Generacionales, SBG, a partir de los precios de cada tipo de SIEFORE generacional, de la siguiente forma:

$$
I_{t}=I_{t-1}\left(\frac{\sum\left(\frac{P_{i t}}{P_{i t-1}}\right)}{n}\right)
$$

en donde: $I_{0}=100, I_{t}=$ Índice el día $t, I_{t-1}=$ Índice el día $t-1, P_{i t}=$ precio de la SIEFORE $i$ el día $t, \mathrm{P}_{i t-1}=$ Precio de la SIEFORE $i$ el día anterior, $n=$ número de sociedades de inversión que cotizaron en cada tipo de SIEFORE. Para calcular el Índice del sistema de las SIEFORE Básicas generacionales, se utilizó el mismo procedimiento, pero esta vez usando el valor diario de cada índice en lugar de los precios diarios.

Dado que la serie es asimétrica y leptocúrtica, como se muestra en los datos estadísticos que se presentan más adelante, se estimó la volatilidad condicional mediante distintos modelos de la familia: GARCH $(1,1)$, EGARCH $(1,1)$ y TARCH (1,1), con el propósito de captar la asimetría. Por último, se simularon con el método de Monte Carlo, el ahorro total acumulado al final de la vida activa de un trabajador tipo, suponiendo diversos niveles de ingresos.

\section{Los modelos GARCH}

En el modelo GARCH, desarrollado por Bollerslev (1986), la varianza condicional depende no sólo de los cuadrados de las perturbaciones, sino también de las varianzas condicionales de periodos anteriores. Así, la varianza condicional del modelo GARCH $(1,1)$ se determina como:

$$
\sigma_{t}^{2}=\alpha_{0}+\alpha_{1} \varepsilon_{t-1}^{2}+\beta \sigma_{t-1}^{2}
$$

\section{Modelo GARCH exponencial}

Por otra parte, en las series financieras es muy frecuente encontrar que las perturbaciones negativas tengan un efecto más pronunciado que las positivas en la volatilidad, este comportamiento asimétrico provocado por perturbaciones externas se puede tratar con un modelo GARCH exponencial o EGARCH, de Nelson (1991):

\footnotetext{
${ }^{9}$ Hasta el 29 de agosto, CONSAR únicamente había publicado los precios de las SIEFORE registrados en Bolsa de cada una de las SIEFORE generacionales hasta el 29 de junio del 2020, razón por la cual sólo se analiza hasta esta fecha el comportamiento de las SIEFORE.
} 


$$
\ln \left(\sigma_{t}^{2}\right)=\alpha_{0}+\alpha_{1}\left|\varepsilon_{t-1} / \sigma_{t-1}\right|+\lambda\left(\varepsilon_{t-1} / \sigma_{t-1}\right)+\beta \ln \left(\sigma_{t-1}^{2}\right)
$$

Esta ecuación muestra la respuesta asimétrica en la volatilidad debido a perturbaciones pasadas positivas o negativas. En general, entre más grande es el choque externo mayor la diferencia en el impacto de la volatilidad. Las tres características que lo distinguen del modelo GARCH son: 1) la ecuación de la varianza condicional está en una forma log-

lineal, lo cual permite que independientemente de la magnitud del $\ln \left(\alpha_{t}^{2}\right)$, el valor implícito de $\alpha_{t}^{2}$ nunca pueda ser negativo; 2) en lugar de usar el valor de $\varepsilon_{t-1}^{2}$, el modelo EGARCH usa un valor estandarizado de $\varepsilon_{t-1}$ (el cual se obtiene al dividir $\varepsilon_{t-1}$ entre $\sigma_{t}$ ), esto permite una interpretación más natural del tamaño y la persistencia de las perturbaciones; 3) y la más importante, es que este modelo permite medir los efectos de apalancamiento. El parámetro $\lambda$ representa el efecto de apalancamiento de $\varepsilon_{t-1} / \sigma_{t-1}$. El modelo presentará asimetría si $\lambda \neq 0$, en la mayoría de las aplicaciones financieras se espera que $\lambda<0$, Tsay (2005).

\section{Modelo TGARCH}

El modelo TGARCH, al igual que el modelo EGARCH, considera los efectos asimétricos en una serie de tiempo. Este modelo fue desarrollado por Rabemananjara y Zakoian (1993), Zakoian (1994) y Glosten Jaganathan y Runke (1993), aunque de manera independiente. La varianza condicional está dada por:

$$
\sigma_{t}^{2}=\alpha_{0}+\alpha_{1} \varepsilon_{t-1}^{2}+\beta \sigma_{t-1}^{2}+\gamma \varepsilon_{t-1}^{2} I_{t-1}
$$

En el modelo $I_{t-1}=1$ si $\varepsilon_{t-1}<0$; de otra forma $I_{t-1}=0$. La condición del efecto de apalancamiento se refleja en $\gamma>0$. La condición para la no-negatividad será $\alpha_{0}>0, \alpha_{1}>0, \beta \geq 0$, y $\alpha_{1}+\gamma \geq 0$, sin embargo, el modelo será válido, aún si $\gamma<0$, siempre y cuando $\alpha_{1}+\gamma \geq 0$, Brooks (2014).

\section{Simulación Monte Carlo}

Este método numérico se atribuye a J. von Neumann y S. Ulam quienes lo diseñaron en 1949. Dado que el método de Monte Carlo permite simular valores de variables aleatorias, se consideró una técnica apropiada para simular el rendimiento y estimar el monto total ahorrado por un trabajador tipo durante su etapa de acumulación, con diferentes niveles de ingreso. Esto permite contar con una base de comparación entre las condiciones actuales del sistema de pensiones y las propuestas de reforma del sistema pensionario que se presentó en septiembre de 2020. Se consideró como variable estocástica los rendimientos del índice de las SIEFORE estimado, y se usó el método de Monte Carlo suponiendo una distribución logarítmica, para calcular el monto total acumulado:

$$
F=\left(\frac{A\left[1-\left(1+\frac{i}{t}\right)^{-n}\left(1+\frac{j}{t}\right)^{n}\right]}{\frac{i}{t}-\frac{j}{t}}\right)(1+i) \quad j \neq i
$$




\section{M. del R. Martínez-Preece y C. Zubieta-Badillo / Contaduría y Administración 65(5) Especial COVID-19, 2020, 1-19 http://dx.doi.org/10.22201/fca.24488410e.2020.3101}

En donde: $F=$ valor futuro de las aportaciones, $A=$ aportaciones trimestrales de los trabajadores, $i=$ tasa de rendimiento anual, $j=$ incremento del salario de los trabajadores, $n=$ número de bimestres durante los cuales se realizarán las aportaciones tripartitas ${ }^{10}, t=$ número de períodos en un $\mathrm{año}^{11}$. Se calcularon los valores futuros de las aportaciones que realizarán trabajadores con tres, cinco, siete y ocho salarios mínimos de ingreso, con el objeto de estimar si la cantidad acumulada al final de su vida laboral será suficiente para adquirir una perpetuidad que les permita obtener una pensión de por lo menos dos salarios mínimos mensuales, de tal forma que no tengan que depender de la pensión mínima garantizada, que actualmente representa 1.3 salarios mínimos. Se realizaron 100,000 iteraciones para cada caso y se supuso un incremento anual del salario mínimo del 5\% anual durante la fase de acumulación.

\section{Resultados}

En esta sección se presentan los índices, los rendimientos y la dinámica de la volatilidad del sistema de pensiones a través de la estimación de la volatilidad condicional. En la última sección se analiza el nivel de aportaciones y el tiempo de cotización que plantea la propuesta de la reforma al sistema de pensiones a la luz de la baja en los ingresos de los trabajadores causada por la crisis.

\section{El comportamiento del sistema de pensiones}

En la Figura 8 se muestran los índices de las SIEFORE y los rendimientos diarios anualizados. En la 8.a) se muestra el comportamiento del índice y los rendimientos de éste hasta noviembre de 2019². En 2008, el sistema de pensiones sufrió su peor caída, con una pérdida del 10\% entre el 2 y el 29 de octubre de ese año. Tuvieron que pasar once meses para que el sistema se recuperara de la crisis, desde que empezaron a manifestarse los primeros síntomas previos a ésta, en mayo de 2008, hasta que volvió al mismo nivel que tenía antes de la crisis, el 30 de abril de 2009. Los rendimientos han presentado amplias fluctuaciones, no sólo las provocadas por la crisis de 2008 y la actual crisis provocada por la pandemia, sino también en 2013. En este año el sistema de pensiones presentó importantes minusvalías debido a alta volatilidad internacional causada, principalmente, por la incertidumbre sobre las decisiones de la Reserva Federal de Estados Unidos respecto al incremento de las tasas de interés. La Figura 8.b) muestra el índice y los rendimientos del sistema con las SIEFORE generacionales, SBG, durante 2020. El índice del sistema con las SIEFORE generacionales presentó una disminución del 8\% a raíz del anuncio de la pandemia en marzo de 2020. El nivel que el índice tenía antes de marzo se alcanzó hasta el 20 de mayo. Los últimos días de mayo y hasta junio de 2020 mantuvo una tendencia al alza. Al cierre de junio de 2020, el sistema recuperó las minusvalías del primer trimestre (CONSAR, 2020a) acumulando plusvalías por 220 mil millones de pesos en comparación con las minusvalías de más de 130 mil millones de pesos que se tuvieron al cierre del primer trimestre.

\footnotetext{
${ }^{10}$ Se ajustaron las semanas de cotización requisito para recibir una pensión a bimestre, ya que es la forma en que los recursos de los trabajadores de depositan en la cuenta individual.

${ }^{11}$ En este caso es de 6, que corresponde al número de bimestres en un año.

${ }^{12}$ En esta fecha CONSAR publicó por última vez el Índice del sistema de la SIEFORE básicas conformado por los cinco tipo de SIEFORES básicas que existían hasta antes de la reestructuración.
} 


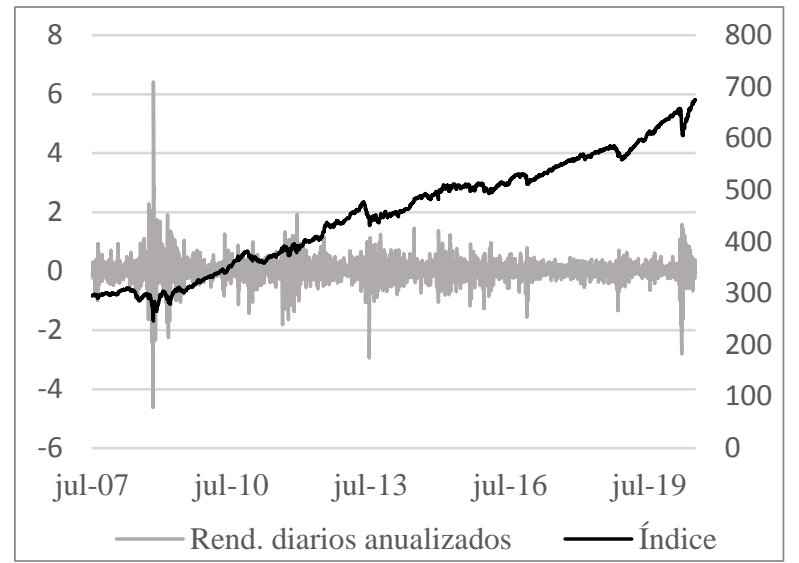

Figura 8.a) Índice del sistema de SIEFORE antes de las SBG (noviembre 2019)

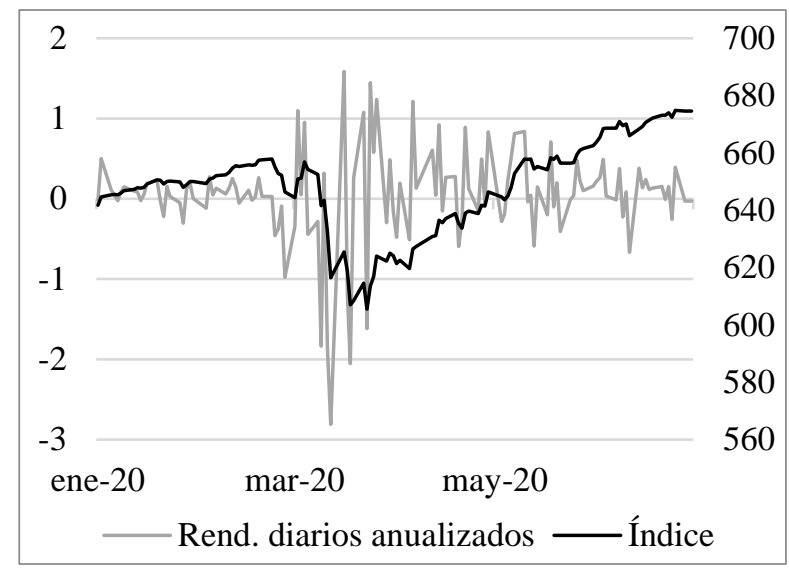

Figura 8.b) Índice del sistema con la creación de las SBG

Figura 8. Índice del sistema de SIEFORE

Fuente: elaboración propia con datos de CONSAR

En la tabla 1 se presentan los datos descriptivos del índice de precios del sistema de las SIEFORES y los rendimientos diarios derivados de éste. Ambas series presentan, sesgo, leptocurtosis, y no siguen una distribución normal.

Tabla 1

Estadísticos descriptivos y efecto ARCH

\begin{tabular}{ccc}
\hline Parámetros & Índice & Rendimientos \\
\hline Media & 354.04 & 0.000252 \\
Mediana & 303.90 & 0.000324 \\
Máximo & 674.82 & 0.059102 \\
Mínimo & 99.55 & -0.042599 \\
Desviación Estándar & 145.09 & 0.0033811 \\
Sesgo & 0.28 & 0.426596 \\
Curtosis & 2.06 & 3.46698 \\
Jarque-Bera & 286.13 & 126571.5 \\
ARCH-LM* & & 795.559 \\
\hline
\end{tabular}

*Resultado de la prueba es el valor de la F- estadística

Fuente: elaboración propia con datos de CONSAR

Se realizó una prueba para detectar heterocedasticidad condicional autorregresiva (ARCH) en los residuos de un modelo ARMA $(1,1)$ aplicado a los rendimientos de los precios. El resultado fue una probabilidad menor al 1\%, lo cual es altamente significativo por lo que se rechaza la hipótesis nula de ausencia de efecto ARCH en la serie, aceptando que la varianza de los errores cambia a través del tiempo, por lo cual la volatilidad se modelará a partir de modelos que permiten incorporar observaciones rezagadas de su varianza.

\section{Volatilidad condicional}

Como se mencionó anteriormente, la volatilidad condicional de los rendimientos se calculó con las primeras diferencias del índice de precios del sistema. Para lo cual, primero se verificó que la serie fuera estacionaria. Los resultados de las pruebas de raíz unitaria: Dickey- Fuller Aumentada, (DFA); Phillip-Perron (PP); y Kwiatkwski-Phillips-Schmidt-Shin (KPPS) se muestran en la Tabla 2. 
M. del R. Martínez-Preece y C. Zubieta-Badillo / Contaduría y Administración 65(5) Especial COVID-19, 2020, 1-19 http://dx.doi.org/10.22201/fca.24488410e.2020.3101

Tabla 2

Pruebas de raíz unitaria

\begin{tabular}{ccccccc}
\hline \multirow{2}{*}{ Variable } & $\begin{array}{c}\text { Estadístico } \\
\text { DFA }\end{array}$ & Valor $p$ & $\begin{array}{c}\text { Valor Crítico } \\
1 \%\end{array}$ & $\begin{array}{c}\text { Valor Crítico } \\
5 \%\end{array}$ & $\begin{array}{c}\text { Valor Crítico } \\
10 \%\end{array}$ & Resultado \\
\hline DFA & -45.43477 & \multirow{2}{*}{0.0001} & -3.432161 & -2.862226 & -2.567179 & Estacionaria \\
PP & -44.59966 & 0.0001 & -3.432161 & -2.862226 & -2.567179 & Estacionaria \\
KPPS & 0.037925 & & 0.739000 & 0.463000 & 0.347000 & Estacionaria \\
\hline
\end{tabular}

Ho: Existe raíz unitaria

Fuente: elaboración propia con datos de CONSAR

Una vez que se verificó que la serie de tiempo de los rendimientos fuera estacionaria, se realizó una prueba de raíz unitaria con quiebre estructural, ya que según se observó en la Figura 11.a) la crisis de 2008 pudo haber causado una ruptura en la serie los rendimientos. En la tabla 3. Se muestran los resultados de la prueba, con la cual se determinó la presencia de un quiebre estructural el 29 de octubre de 2008, a raíz de la crisis financiera de ese año.

Tabla 3

Prueba de raíz unitaria con quiebre estructural

\begin{tabular}{llll}
\hline & & Estadístico-t & Valor $p$ \\
\hline Estadístico de la prueba DFA & & -48.6678 & $<0.01$ \\
Valores críticos & al 1\% & -5.347598 & \\
& al 5\% & -4.859812 & \\
& al 10\% & -4.607324 & \\
Fecha del quiebre estructural: & & 29 de octubre de 2008 & \\
\hline
\end{tabular}

$\mathrm{H}_{0}$ : Existe raíz unitaria con rompimiento estructural tanto en la tendencia como en el intercepto de la serie Fuente: Elaboración propia con datos de CONSAR

Tabla 4

Resultados de los modelos de la familia GARCH utilizados

\begin{tabular}{|c|c|c|c|c|c|c|c|c|}
\hline \multicolumn{3}{|c|}{ Modelo GARCH 1,1 } & \multicolumn{3}{|c|}{ Modelo EGARCH 1,1 } & \multicolumn{3}{|c|}{ Modelo TARCH 1,1 } \\
\hline & Coeficiente & Valor-P & & Coeficiente & Valor-P & & Coeficiente & Valor-P \\
\hline M & 0.000362 & 0.00000 & M & 0.000294 & 0.00000 & M & 0.000293 & 0.0000 \\
\hline$\alpha_{0}$ & 4.07E-07 & 0.00000 & $\alpha_{0}$ & -0.572436 & 0.00000 & $\alpha_{0}$ & $3.37 \mathrm{E}-07$ & 0.0000 \\
\hline$\alpha_{1}$ & 0.184047 & 0.00000 & $\alpha_{1}$ & 0.283139 & 0.00000 & $\alpha_{1}$ & 0.086799 & 0.0000 \\
\hline \multirow[t]{2}{*}{$\beta_{1}$} & 0.805527 & 0.00000 & $\lambda$ & -0.101245 & 0.00000 & $\gamma$ & 0.149511 & 0.0000 \\
\hline & & & $\beta_{1}$ & 0.967832 & 0.00000 & $\beta_{1}$ & 0.824451 & 0.0000 \\
\hline Dum & $-1.81 \mathrm{E}-07$ & 0.01100 & Dum & -0.028525 & 0.00090 & Dum & $-1.17 \mathrm{E}-07$ & 0.0631 \\
\hline \multicolumn{9}{|c|}{ Criterios de información } \\
\hline AIC & \multicolumn{2}{|c|}{-8.963633} & \multicolumn{3}{|c|}{-8.982865} & \multicolumn{3}{|c|}{-8.980162} \\
\hline Schwarz & \multicolumn{2}{|c|}{-8.954317} & \multicolumn{3}{|c|}{-8.971686} & \multicolumn{3}{|c|}{-8.968983} \\
\hline H-Q & \multicolumn{2}{|c|}{-8.960297} & \multicolumn{3}{|c|}{-8.978862} & \multicolumn{3}{|c|}{-8.976158} \\
\hline ARCH-LM* & \multicolumn{2}{|c|}{0.797700} & \multicolumn{3}{|c|}{0.953500} & \multicolumn{3}{|c|}{0.617100} \\
\hline
\end{tabular}

*Los valores se refieren a probabilidad de F.

Fuente: elaboración propia con los datos de la estimación ${ }^{13}$

${ }^{13}$ Las estimaciones de los modelos GARCH se realizaron con E-Views 11. 
Para incorporar el quiebre estructural detectado en los modelos GARCH utilizados se incorporó una variable dummy. Dado que los parámetros en los datos estadísticos descriptivos muestran la presencia de asimetría se utilizaron modelos GARCH $(1,1)$, EGARCH $(1,1)$ y TARCH $(1,1)$, éstos dos últimos captan los efectos de asimetría que se pudieran presentar en la volatilidad condicional. En la Tabla 4 se presentan los resultados.

La variable dummy resultó significativa al $10 \%$ en los tres modelos, sin embargo, para el modelo EGARCH resultó altamente significativa con una probabilidad de menos de 1\%. Los resultados de la prueba ARCH-LM permiten aceptar la hipótesis nula de ausencia de efecto $\mathrm{ARCH}$, ya que en todos los casos la probabilidad asociada es mayor al $10 \%$, por lo que se puede decir que al no quedar efectos $\mathrm{ARCH}$ en los residuos estandarizados, la varianza se especificó correctamente. El modelo EGARCH es el que presenta la menor probabilidad de tener efectos ARCH. Por otra parte, aunque los criterios de información son muy similares, el modelo EGARCH es el que presenta una menor pérdida de información.

Los modelos GARCH y TARCH muestran una alta persistencia de la volatilidad, lo cual se observa en la suma de los coeficientes $\alpha_{1}$ y $\beta_{1}$ muy cercanos a 1 , sobre todo el modelo GARCH $(1,1)$. El efecto de asimetría se capturó mediante los modelos EGARCH $(1,1)$ y TARCH $(1,1)$. En el caso del modelo EGARCH $(1,1)$, el coeficiente $\lambda$ es negativo, lo cual significa que las perturbaciones negativas tienen un impacto mayor que choques positivos de la misma magnitud. En el modelo TARCH el coeficiente $\gamma$ es positivo, lo cual, de acuerdo con la especificación de este modelo, también significa que el efecto de las perturbaciones negativas es mayor que las positivas del mismo tamaño. Con estos resultados se encontró que existe persistencia de la volatilidad y que las perturbaciones negativas tienen un mayor impacto que las positivas.

En la Figura 9, se muestra la gráfica de la volatilidad condicional que se obtuvo del modelo EGARCH $(1,1)$. Aquí se presenta la volatilidad condicional representada por la desviación estándar condicional diaria. El mayor incremento en la volatilidad condicional se presentó durante la crisis de 2008, seguido del aumento en la volatilidad provocada por la crisis del COVID, lo que muestra el impacto de la actual crisis con respecto al riesgo en el que ha incurrido el sistema de pensiones desde 2008. El pico que se aparece en 2013, representa la inestabilidad del sistema durante ese año.

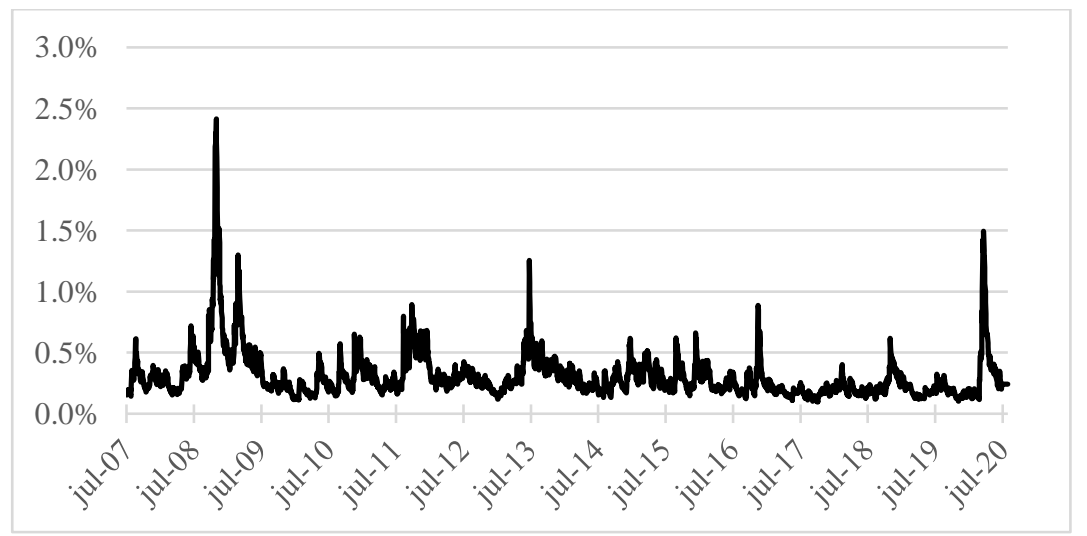

Figura 9. Volatilidad condicional del sistema de pensiones Fuente: elaboración propia con los resultados de la estimación

\section{Efectos de la crisis en el sistema de pensiones y la propuesta de reforma del sistema}

Un efecto directo de la crisis, y quizá el que más impacto tenga en el monto de las pensiones que recibirán los trabajadores es el desempleo. A pesar del crecimiento en los recursos manejados por las Administradoras de los Fondos para el Retiro, 


\section{M. del R. Martínez-Preece y C. Zubieta-Badillo / Contaduría y Administración 65(5) Especial COVID-19, 2020, 1-19 http://dx.doi.org/10.22201/fca.24488410e.2020.3101}

AFORE, en el segundo trimestre de 2020, la CONSAR reportó un incremento en los retiros parciales por desempleo ${ }^{14}$ de la cuentas de los trabajadores, al cierre de junio de 2020, de $\$ 8570$ millones de pesos (CONSAR, 2020b) cifra superior en $37.52 \%$ a lo reportado en el mismo mes con respecto al año pasado.

El desempleo ha provocado que los trabajadores hagan uso parcial de los recursos acumulados en sus cuentas individuales para sobrellevar la crisis económica. Esto tendrá el efecto de disminuir al ahorro total acumulado para su pensión. Este elemento que, además de tener consecuencias negativas directas para aquellos trabajadores que utilicen este beneficio, agrega presión al sistema, debido a que su sustentabilidad ha sido cuestionada en diversas ocasiones, como se mencionó anteriormente. La propuesta de reforma del sistema incluye medidas que pretenden incrementar el nivel de ahorro total, por lo tanto, su aplicación pudiera resultar muy conveniente en estos momentos, si los cambios propuestos tienen el potencial de revertir algunos de los efectos negativos de la crisis en el total de recursos que podrán acumular los trabajadores.

\section{La propuesta de reforma ante la crisis por COVID-19}

El 29 de septiembre se sometió ante la Cámara de Diputados una propuesta de modificación del sistema de pensiones mediante la reforma, adición y derogación de diversas disposiciones de la Ley del Seguro Social y de la Ley de los Sistemas de Ahorro para el Retiro. Entre los puntos más importantes se encuentran: el incremento en el monto de las aportaciones, la disminución de las comisiones, la reducción de las semanas de cotización, y el hecho que trabajadores no afiliados al IMSS o al ISSSTE puedan abrir una cuenta individual destinada a su pensión.

Dado que los retiros por desempleo harán que el saldo total del trabajador disminuya, al igual que el número de semanas cotizadas, mismas que serán restadas del tiempo de cotización acumulado de manera proporcional al retiro efectuado, como se mencionó anteriormente, se decidió analizar dos puntos esenciales para que el trabajador logre acumular los recursos suficientes para obtener una pensión arriba de un nivel de subsistencia. Estos dos puntos son: las aportaciones y el tiempo de cotización.

En lo que respecta a las aportaciones tripartitas la reforma propone incrementarlas del $6.5 \%$ al $15 \%$ aumentando la aportación del patrón del $5.15 \%$ a un $13.7 \%$ conforme a una tabla con los porcentajes aplicables de acuerdo con el salario del trabajador; y la aportación del estado tendiente a apoyar a los trabajadores con menores ingresos iniciando en 2023 hasta alcanzar los topes máximos en 2030, también conforme a una tabla de referencia. Sin duda, un incremento en las aportaciones elevará la suma acumulada al final de la vida activa del trabajador, sin embargo, se debe considerar también el efecto de reducir las semanas de cotización de los trabajadores. La reforma contempla reducir éstas de 1250 a 750, mismas que se irán incrementando hasta llegar a 1000 en el 2031.

Para poder determinar el efecto en el total acumulado al final del período de acumulación, se plantearon tres escenarios. En el primero se tomó el caso de continuar cotizando con el esquema actual. En el segundo se incluirá un incremento en las aportaciones. En un tercer escenario se incrementarán en las aportaciones, pero se reducirá el número de semanas de cotización a 750 .

Como se explicó en la metodología, se simuló con el método de Monte Carlo el ahorro total acumulado por los trabajadores bajo los tres escenarios descritos anteriormente. Se consideró el rendimiento como la variable aleatoria y se supuso un incremento salarial del $5 \%$ anual $^{15}$ durante la vida activa del trabajador ${ }^{16}$ en todos los escenarios. En la Tabla 5 se muestra cuánto se podría ahorrar bajo el esquema actual suponiendo aportaciones totales del $6.5 \%$ del salario del

\footnotetext{
14 Este beneficio está disponible para trabajadores con al menos 46 días de desempleo, y no hayan efectuado ningún retiro en los últimos cinco años, (CONSAR, 2020)

${ }^{15}$ Estos incrementos se incorporaron a la simulación bimestralmente, considerando una tasa equivalente del $5 \%$ anual.

${ }^{16}$ Se considera como vida activa del trabajador 1250 semanas de cotización bajo el escenario 1 y 2 o 750 bajo el escenario 3 .
} 


\section{M. del R. Martínez-Preece y C. Zubieta-Badillo / Contaduría y Administración 65(5) Especial COVID-19, 2020, 1-19 http://dx.doi.org/10.22201/fca.24488410e.2020.3101}

trabajador, concluyéndose que difícilmente aquellos trabajadores que ganen menos de 8 salarios mínimos estarán en condiciones de comprar una perpetuidad que les ofrezca una pensión de dos salarios mínimos mensuales o más, situación que les posibilitaría el no tener que depender de la pensión mínima garantizada de 1.3 salarios mínimos ${ }^{17}$. En el segundo escenario se supuso un incremento en la aportaciones del 15\% anual, en este caso los trabajadores con poco más de tres salarios mínimos estarían en condiciones de recibir pensiones arriba de la mínima garantizada. El tercer escenario consistió en suponer el incremento en las aportaciones, pero con una reducción del plazo de cotización de 1250 semanas a 750, como lo indica en un primer momento la propuesta de reforma. En este caso el trabajador estaría ligeramente peor que sin reforma, el trabajador tendría que laborar durante menos años, pero al final tendría que depender de la pensión mínima garantizada.

Tabla 5

Montos acumulados al final de la vida activa del trabajador

\begin{tabular}{|c|c|c|c|c|}
\hline I.Sin reforma & $3 \mathrm{sm} *$ & $5 \mathrm{sm}$ & $7 \mathrm{sm}$ & $8 \mathrm{sm}$ \\
\hline $90 \%$ probabilidad & $\begin{array}{c}834026 \\
749829-927 \\
033 \\
\end{array}$ & $\begin{array}{c}1390043 \\
1249715-1 \\
545055 \\
\end{array}$ & $\begin{array}{c}1946060 \\
1749600-2 \\
163077 \\
\end{array}$ & $\begin{array}{c}2224068 \\
1999543-2 \\
472088 \\
\end{array}$ \\
\hline $\begin{array}{l}\text { II. Con reforma: aumento aportación, mismo } \\
\text { tiempo de cotización }\end{array}$ & $3 \mathrm{sm}$ & $5 \mathrm{sm}$ & $7 \mathrm{sm}$ & $8 \mathrm{sm}$ \\
\hline $90 \%$ probabilidad & $\begin{array}{l}1924674 \\
1730374-2 \\
13937 \\
\end{array}$ & $\begin{array}{c}3207791 \\
2883956-3565 \\
511 \\
\end{array}$ & $\begin{array}{c}4490906 \\
4037539-4991 \\
715 \\
\end{array}$ & $\begin{array}{c}5132465 \\
4614330-5704 \\
817 \\
\end{array}$ \\
\hline $\begin{array}{l}\text { III. Con reforma: aumento aportación, menos } \\
\text { tiempo de cotización }\end{array}$ & $3 \mathrm{sm}$ & $5 \mathrm{sm}$ & $7 \mathrm{sm}$ & $8 \mathrm{sm}$ \\
\hline $90 \%$ probabilidad & $\begin{array}{c}705276 \\
661494-752 \\
711\end{array}$ & $\begin{array}{c}1175460 \\
1102490-1 \\
254519\end{array}$ & $\begin{array}{c}1645645 \\
1543485-1 \\
756326\end{array}$ & $\begin{array}{c}1877904 \\
1764983-2 \\
007230\end{array}$ \\
\hline
\end{tabular}

* sm iniciales de salario mínimo

Fuente: elaboración propia con los datos de la simulación

Ante la situación actual, sería conveniente volver a analizar los puntos que incluye la reforma. El mejor escenario es el segundo, mantener las 1250 semanas de cotización junto con un incremento en las aportaciones, esta combinación traería como consecuencia un incremento significativo en el monto total acumulado. Esta situación beneficiaría a todos los trabajadores adscritos al sistema de pensiones, pero ayudaría en especial a aquellos trabajadores que realizaron retiros anticipados y que han dejado de cotizar a raíz del desempleo. De esta forma se contaría con un sistema de pensiones estructurado de tal forma que ayudara a compensar los montos que se dejarían de ahorrar debido a la crisis.

\section{Conclusiones}

Se hizo una revisión del entorno económico y de los principales efectos de la crisis en éste. Se expuso la forma en que quedaron constituidos las nuevas SIEFORE generacionales ante la reestructuración del régimen de inversión del sistema de pensiones y se presentó el comportamiento de los precios y la volatilidad del sistema desde julio de 2007 hasta junio de 2020, abarcando la crisis de 2008 y la actual crisis provocada por la pandemia. El riesgo del sistema pensionario se analizó con respecto a la dinámica de la volatilidad condicional, detectándose que existe persistencia de la volatilidad y un efecto de asimetría que provoca que las perturbaciones negativas tengan un impacto mayor que aquellas positivas, con lo

\footnotetext{
${ }^{17}$ La reforma no deroga la pensión universal para adultos mayores, que serviría de complemento a lo que se obtenga de pensión por parte de sistema de ahorro para el retiro o a la pensión mínima garantizada.
} 
cual el comportamiento del sistema se ve afectado ante movimientos negativos externos. Este efecto se presentó no obstante que el $68.9 \%$ de los instrumentos financieras incluidos en los portafolios de inversión son instrumentos de deuda, y de este, poco más del 50\% representa deuda gubernamental. Sin embargo, lo anterior no significa necesariamente que haya aumentado el riesgo del sistema en general, descartándose la primera hipótesis. El sistema obtuvo, a septiembre de 2020, una rápida recuperación.

Por otra parte, también se mostró que el desempleo causado por la crisis, provocó retiros anticipados, por lo que se simuló el total acumulado durante la vida activa del trabajador para determinar si los efectos negativos de la crisis pueden compensarse mediante los cambios en la estructura del sistema de pensiones que propone la reforma del sistema pensionario. Se analizó el efecto del incremento en las aportaciones y la reducción del plazo, concluyéndose que es importante incrementar las aportaciones, sin embargo, no es deseable reducir el plazo de cotización para evitar anular el efecto positivo del incremento en las aportaciones sobre el total acumulado. Este punto es importante desde distintas ópticas. Para los trabajadores, resulta obvio que la posibilidad de acumular mayores saldos totales al final de su vida activa, les permitirán niveles de consumo por encima del nivel de subsistencia, lo cual es el objetivo final del actual sistema de pensiones. Desde la perspectiva fiscal, el realizar reformas adecuadas que permitan a los trabajadores recibir una pensión por encima de la mínima garantizada reducirá la presión fiscal, al existir un mayor número de trabajadores que logren comprar una perpetuidad y que no dependan de la pensión mínima garantizada que ofrece el gobierno.

Considerando lo anterior, se presentan nuevas líneas de investigación. En especial respecto al desempeño que tendrá la política de inversión ante la creación de las SIEFORE generacionales, ya que el nuevo régimen de inversión permite la incorporación de una proporción mayor de instrumentos de deuda para los trabajadores más jóvenes. Asimismo, es importante analizar detalladamente cada punto de la reforma del sistema de pensiones, considerando que la crisis añade elementos que también se deben considerar. El impacto que ha tenido la crisis en los niveles de ocupación, produce un incremento en la discontinuidad de las trayectorias laborales, y cambios en la densidad de cotización, mismos que deben considerarse para poder evaluar el sistema de pensiones bajo la perspectiva de su sustentabilidad y sostenibilidad.

\section{Referencias}

Banco de México, (2020). Información estadística. Disponible en: https://www.banxico.org.mx/SieInternet/consultarDirectorioInternetAction.do?accion=consultarCuadro\&idCua dro=CF57. Consultado el 29 de Agosto de 2020.

Bollerslev, Tim (1986), Generalized Autoregressive Conditional Heteroskedasticity, Journal of Econometrics, 31, pp. 307-327. https://doi.org/10.1016/0304-4076(86)90063-1

Brooks Chris, (2014). Introductory Econometrics for Finance. Cambridge University Press.

CONSAR, Comisión Nacional del Sistema de Ahorro para el Retiro, (2020a) Informe al Congreso. CONSAR. Disponible en: https://www.gob.mx/consar/documentos/informes-trimestrales-de-la-consar, consultado 15 septiembre 2020.

CONSAR, Comisión Nacional del Sistema de Ahorro para el Retiro (2020b). Retiro Parcial por desempleo. IMSS, ISSSTE. Blog CONSAR. Disponble en: https://www.gob.mx/consar/articulos/retiro-parcial-por-desempleo239845? idiom=es, consultado 18 de septiembre 2020

CONSAR, Comisión Nacional del Sistema de Ahorro para el Retiro, (2020c). Precios de Bolsa de las SIEFORE. https://datos.gob.mx/busca/dataset/listado-de-los-precios-de-bolsa-de-las-siefores-por-tipo-de-fondo-deinversion. Consultado el 27 de agosto de 2020

De la Torre-Torres de la O.V., Galeana-Figueroa E., Martínez Torre Enciso M.I, Aguilasocho-Montoya D. (2015a). A minimum variance benchmark to measure the performance of pension funds in Mexico. Contaduría $y$ Administración. Vo. 50, No. 3, pp.593-610. http://dx.doi.org/10.22201/fca.24488410e.2021.2327

De la Torre-Torres de la, O.V., Galeana-Figueroa E., Aguilasocho-Montoya D. (2015b). An Actual Position Benchmark for Mexican Pension Funds Performance. Economía, Teoría y Práctica, No. 43, pp.133-154. https://doi.org/10.24275/ETYPUAM/NE/432015/DelaTorre 
De la Torre-Torres de la O.V., Galeana-Figueroa E., Aguilasocho-Montoya D. (2020a). A Two-Regime Performance Test of the Mexican Public Pension Funds (fsas). In: Peris-Ortiz M., Álvarez-García J., Domínguez-Fabián I., Devolder P. (eds) Economic Challenges of Pension Systems. Springer, Cham. https://doi.org/10.1007/978-3030-37912-4_17

Encinas-Goenechea B., Meneu-Gaya R., de la Cruz del Río-Rama M. (2020) The Public Pension Systems and the Economic Crisis. In: Peris-Ortiz M., Álvarez-García J., Domínguez-Fabián I., Devolder P. (eds) Economic Challenges of Pension Systems. Springer, Cham. https://doi.org/10.1007/978-3-030-37912-4_3

Glosten, L.R., Jaganathan R., y Runkle D. (1993). On the Relation between the Expected Value and the Volatility of the Normal Excess Return on Stocks, Journal of Finance, 48, 1779-1801. https://doi.org/10.1111/j.15406261.1993.tb05128.x

FMI, Fondo Monetario Internacional (2020). Una crisis como ninguna otra, una recuperación incierta. Actualización de las perspectivas de la economía mundial. Disponible en: https://www.imf.org/es/Publications/WEO/Issues/2020/06/24/WEOUpdateJune2020. Consultado el 10 de agosto de 2020.

Instituto Nacional de Estadística, Geografía e Informática. INEGI, (2020). Datos. Disponible en: https://www.inegi.org.mx/temas/pib/. Consultado el 3 de septiembre de 2020.

Lachman Jack, (2013). Pension: It's Impact on the World Economy. International Journal of Business and Commerce, Vol. 3, No.2, pp. 14-25.

López-Herrera F., Martínez-Preece M.R., Santillán-Salgado R.J. (2020). Regime-Switching in the Volatility of Mexican Pension Fund Returns. In: Peris-Ortiz M., Álvarez-García J., Domínguez-Fabián I., Devolder P. (eds) Economic Challenges of Pension Systems. Springer, Cham. https://doi.org/10.1007/978-3-030-37912-4_18

Martínez-Preece Marissa R., Sosa Castro Miriam y Zubieta Badillo Carlos (2019). Dinámica y desempeño de los fondos de pensión en México (1997-2018): un análisis de volatilidad condicional con cambios estructurales. Revista de Economía. Vol. XXXVI- Núm 93. pp 9-34. https://doi.org/10.33937/reveco.2019.104

Nelson, D. B. (1991). Conditional Heteroskedasticity in Asset Returns. A New Approach. Econometrica, Vol. 59, No. 2, pp. 347-370. https://doi.org/10.2307/2938260

Nuñez Mora José Antonio y León Alvarado Martha Angélica (2019). Determinación de un portafolio de referencia para las SIEFORE Básicas a través de un modelo de riesgo-rendimiento que optimiza la tasa de reemplazo. EconoQuantum. https://doi.org/10.18381/eq.v16i1.7159

OECD, (2016), OECD Reviews on pension systems: Mexico.OECD Publishing. Paris. DOI: http://dx.doi.org/10.1787/97892642455939-1-en. Disponible en: http://www.oecd.org/mexico/oecd-reviews-ofpension-systems-mexico-9789264245938-en.htm. Consultado el 18 de noviembre de 2017

OCDE, (2020a). Perspectivas económicas de la OCDE. Volumen 2020, Número 1. Editorial OCDE, Paris, https://doi.org/10.1787/0d1d1e2e-en. Disponible en http://oecd.org/perspectivas-economicas/. Consultado el 6 de agosto de 2020.

OECD, (2020b), OECE Pensions in figures. Disponible en: https://www.oecd.org/daf/fin/private-pensions/Pension-Fundsin-Figures-2020.pdf. Consultado en el 25 de agosto de 2020

Ortiz Isabel, Durán-Valverde Favio, Urban Stefan, Wodsak Veronika, Yu Zhiming (2019). La reversión de la privatización de las pensiones: Reconstruyendo los sistema públicos de pensiones en los países de Europa Oriental y América Latina (2000-2018). Documento de Trabajo No. 63, Departamento de Protección Social, Oficina Internacional del Trabajo.

Rabemananjara, R., \& Zakoïan, J.M. (1993). Threshold ARCH models and asymmetries in volatility. Journal of Applied Econometrics, 8, 31-49. https://doi.org/10.1002/jae.3950080104

Ramírez Berenice (2019). La necesaria construcción de un sistema público de pensiones para la sociedad mexicana. El Trimestre Económico. Vol. LXXXVI (4). Número 334. pp.967-1001. https://doi.org/10.20430/ete.v86i344.982

Tsay Ruey S. (2005), Analysis of Financial Time Series, $2^{\text {nd }}$ edition, Wiley-Interscience. Nueva Jersey, Estados Unidos.

UNCTAD (2020). United Nations Conference on Trade and Development. Disponible en: https://unctad.org/en/PublicationsLibrary/ditcinf2020d3_en.pdf. Consultado el 5 de septiembre de 2020.

Zakoïan, J. M. (1994). Threshold heteroskedastic models. Journal of Economic Dynamics and Control, 18, $931-955$. https://doi.org/10.1016/0165-1889(94)90039-6Get rights and content 